\title{
Finding Regressions in Projects under Version Control Systems
}

\author{
Jaroslav Bendík, Nikola Beneš, and Ivana Černá \\ Faculty of Informatics, Masaryk University, Brno, Czech Republic \\ \{xbendik, xbenes3, cerna\}@fi.muni.cz
}

\begin{abstract}
Version Control Systems (VCS) are frequently used to support development of large-scale software projects. A typical VCS repository of a large project can contain various intertwined branches consisting of a large number of commits. If some kind of unwanted behaviour (e.g. a bug in the code) is found in the project, it is desirable to find the commit that introduced it. Such commit is called a regression point. There are two main issues regarding the regression points. First, detecting whether the project after a certain commit is correct can be very expensive as it may include large-scale testing and/or some other forms of verification. It is thus desirable to minimise the number of such queries. Second, there can be several regression points preceding the actual commit; perhaps a bug was introduced in a certain commit, inadvertently fixed several commits later, and then reintroduced in a yet later commit. In order to fix the actual commit it is usually desirable to find the latest regression point.

The currently used distributed VCS contain methods for regression identification, see e.g. the git bisect tool. In this paper, we present a new regression identification algorithm that outperforms the current tools by decreasing the number of validity queries. At the same time, our algorithm tends to find the latest regression points which is a feature that is missing in the state-of-the-art algorithms. The paper provides an experimental evaluation of the proposed algorithm and compares it to the state-of-the-art tool git bisect on a real data set.
\end{abstract}

\section{Introduction}

Version Control Systems (VCS) have become ubiquitous in the area of (not only) software development, from small toy projects to large-scale industrial ones. The recent years saw a rise in the popularity of Distributed VCS such as git [8], bazaar [4, Mercurial 14] and many others. These allow for almost seamless cooperation of a large number of developers and support extensive project branching and merging of branches. After a project has been in the development process for some time, the commit graph of its repository may grow to be very large.

As projects grow larger, the appearance of bugs (i.e. unwanted behaviour of the developed product) is going to be inevitable. Software bugs can be usually caught 
early if the development teams employ extensive testing techniques (unit tests, performance regression tests, etc.); however, from time to time a bug, or a commit that changed properties of the project, may creep into the VCS repository and lie there undetected for some time. Such bug is usually discovered by e.g. extending the coverage of the tests or by employing some other verification technique such as model checking [5]. In order to fix the bug it is very useful to identify the commit that introduced the bug as this commit typically contains a relatively small set of source code changes. It is much easier to properly understand and fix a bug when you only need to check a very small set of changes of the source code. Sometimes we are not looking for the commit that introduced a bug, but rather for a commit that caused a change between some "old" and "new" state of the project. As an example, we might be looking for the commit that introduced a particular fix. In such cases it can seem confusing to use the terms "correct" and "buggy" to refer to the state before and after the change, respectively. We thus instead use the terms valid and invalid commit; we further use the term regression point to denote the point where the property of interest has been changed.

The problem of finding regression points has been addressed before and there have been developed tools for solving this problem, such as git bisect [10. These tools have proved themselves to be very useful and are commonly used during software development nowadays. Yet, there are several issues related to finding regression points and only some of them are targeted by the state-of-the-art tools.

First, the search for regression points consists of several queries of the form: "Given a certain commit, is the bug present in the system after this commit?" Such queries, which we call validity queries, may consist of several expensive tasks like running tests, model checking, code inspection, or other forms of verification. It is thus desirable to minimise the number of these queries. Second, the validity of commits does not, in general, have to be monotone. Perhaps a bug was introduced in a certain commit, inadvertently fixed several commits later, and then reintroduced in a yet later commit. This means that there are possibly several regression points preceding the actual invalid commit, but only the identification of the latest regression point can usually help us to fix the bug. The third issue concerns large projects with many branches. If, for example, a new test case is employed, then more than just one active branch can fail the test case and it is desirable to identify a regression point for each of these branches. We can find the regression point for each branch separately. However, dealing with all branches simultaneously can save some validity queries and thus optimise the search.

The state-of-the-art tools target the need to minimise the number of validity queries that are performed. However, they do not tend towards identification of latest regression points, and they deal with only a single invalid branch at a time.

The goal of this paper is to provide a novel algorithm, called the Regression Predecessors Algorithm (RPA), that solves the problem of finding regression points in VCS repositories. The algorithm minimises the number of validity queries and at the same time tries to find the latest regression points both for 
single and multiple invalid branches. RPA has several variants which we compare on a set of real open-source projects. Moreover, we compare the RPA algorithm with the state-of-the-art tool git bisect and demonstrate that our algorithm outperforms git bisect in all of the three above-mentioned criteria: in the number of performed validity queries, in finding the latest regression points, and in finding regression points for multiple invalid branches.

The rest of the paper is organised as follows. Section 2 defines basic notions and states the problem formally. Section 3 presents the Regression Predecessors Algorithm and illustrates its behaviour on a small example. Section 3.5 reviews the related work and compares RPA with other known algorithms. Section 4 gives an experimental evaluation of different variants of RPA and compares RPA with the state-of-the-art tool git bisect on a set of real benchmarks.

\section{Preliminaries and Problem Formulation}

Definition 1. A rooted directed acyclic graph is a directed graph $G=(V, E)$ with exactly one root (i.e. a vertex with no incoming edges) and with no cycle (i.e. there is no path $\left\langle v_{0}, v_{1}, \cdots v_{k}\right\rangle$ in the graph such that $v_{0}=v_{k}$ and $k>0$ ). A rooted annotated directed acyclic graph (RADAG) is a pair $(G$, valid), where $G$ is a rooted directed acyclic graph with root $r$ and valid $: V \rightarrow$ Bool is a validation function satisfying valid $(r)=$ True.

We use RADAGs to model structures which arise from using version control systems (VCS). Each vertex in $G$ corresponds to a commit in VCS repository. An edge between two vertices represents two subsequent commits. The root corresponds to the initial commit and the leaves (vertices with no outgoing edges) correspond to the latest commits of individual branches.

The validation function expresses whether a particular commit has the desired property that the system after this commit is correct (i.e. does not contain the bug). We call the vertices with this property (for which the validation function evaluates to True) valid vertices and the others invalid ones. Note that we assume that the graph has only one root and that the root is valid. If this is not the case, the graph can be easily modified by adding a dummy initial valid commit.

Definition 2. A regression point of a $R A D A G((V, E)$, valid $)$ is a pair of vertices $(u, v)$ such that $(u, v) \in E$, valid $(u)=$ True, and valid $(v)=$ False. A regression point $(u, v)$ is a regression predecessor of a vertex $w \in V$ if $w$ is equal to $v$ or $w$ is reachable from $v$.

We are now ready to formally state our problem.

Regression Predecessors Problem: Given a RADAG $(G$, valid) and a set of invalid leaves $L$ of $G$, find at least one regression predecessor for each leaf from $L$.

Note that one invalid leaf can have several regression predecessors and one regression point can be a regression predecessor of several invalid leaves. Therefore, the regression predecessors problem may have several different solutions. 


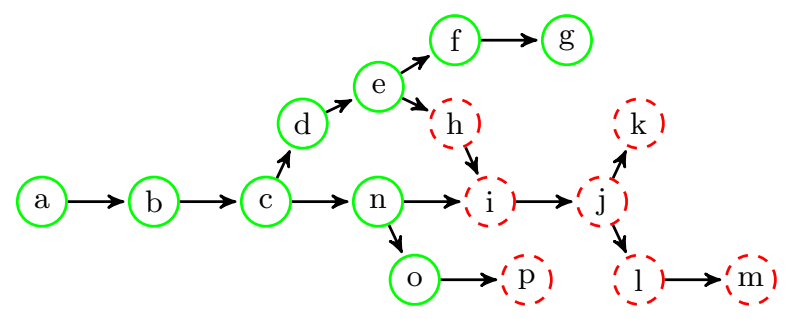

Fig. 1. An example of a RADAG, the dashed vertices are invalid. There are three invalid leaves in this example: $k, m, p$, and four regression points: $(c, d),(e, h),(n, i)$ and $(o, p)$. The first three regression points are regression predecessors of invalid leaves $k, m$; the fourth regression point is the regression predecessor of the invalid leaf $p$.

\section{Regression Predecessors Algorithm (RPA)}

A naive solution to the regression predecessors problem would be to evaluate the function valid for each vertex, identify all regression points in $G$, and find a regression predecessor of each invalid leaf using the reachability relation. Because this approach identifies all regression points we can choose the latest regression predecessor of every invalid leaf. However, the price is crucial; the function valid is evaluated for every vertex which is assumed to be extremely time-consuming.

In this section we present a new algorithm, the Regression Predecessors Algorithm (RPA), that substantially decreases the number of vertices for which the function valid is evaluated and tends to find the latest regression points at the same time.

\subsection{Basic Schema}

The main idea of RPA is based on the observation that if a leaf $l$ is invalid then every path starting in a valid vertex and leading to $l$ must contain at least one regression predecessor of $l$. This reduces the problem to two tasks: finding a path and detecting a regression point on the path.

For the basic description of RPA see Algorithm 1. The algorithm maintains the set UnprocessedLeaves which consists of those invalid leaves for which a regression predecessor has not been computed yet. The set KnownValid consists of those vertices for which the function valid has been evaluated and are valid (initially only the root is known to be valid). In each iteration, the algorithm chooses a leaf $l$ from the set UnprocessedLeaves. A regression predecessor of $l$ is acquired by building a path $p_{l}$ which connects a valid vertex $x \in$ Known Valid with $l$ and by finding a regression point $(u, v)$ on this path. While searching for the regression point $(u, v)$, the function valid is evaluated for some vertices on the path $p_{l}$. The newly detected valid vertices form a set NewValid and the set Known Valid is updated accordingly.

The algorithm also exploits the fact that one regression point can be a regression predecessor of several invalid leaves. Therefore, every time a regression 


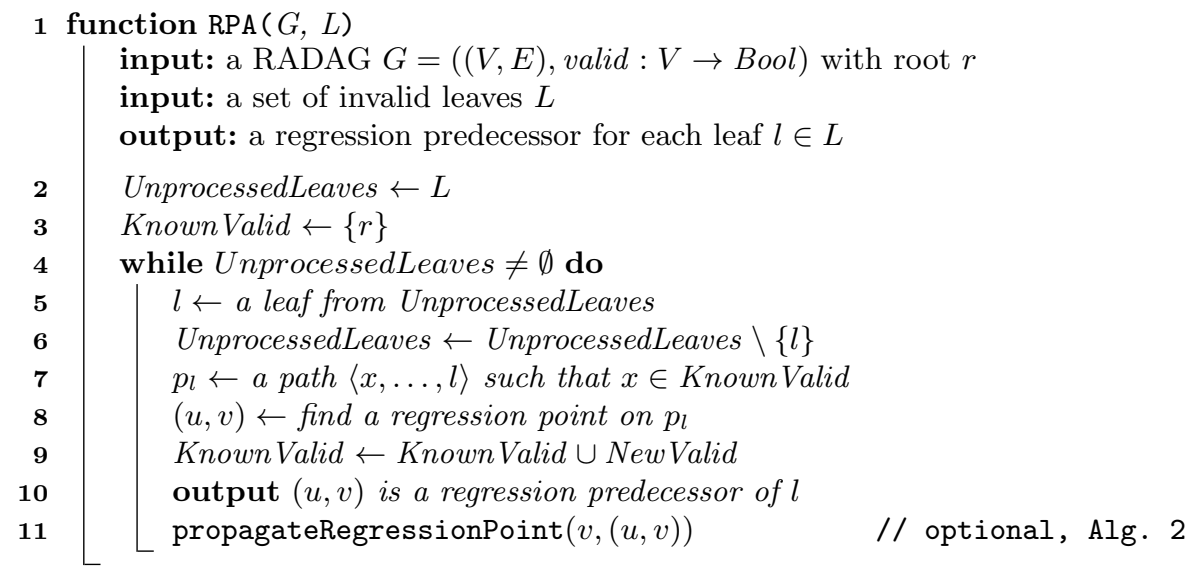

Algorithm 1: Regression Predecessors Algorithm (basic schema)

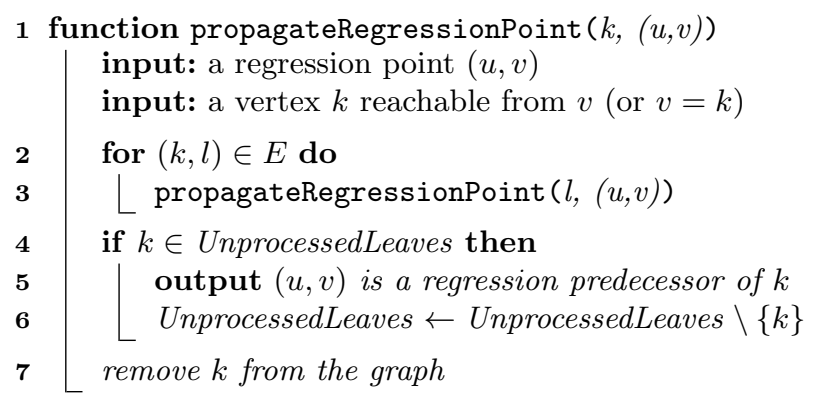

Algorithm 2: regression point propagation

point $(u, v)$ is found, it is propagated to every invalid leaf $m \in$ UnprocessedLeaves such that $m$ reachable from $v$. Every such $m$ is removed from UnprocessedLeaves (see Algorithm 2). After this propagation step, the procedure also removes from the graph all vertices reachable from $v$. By removing vertices we avoid propagation of regression points to leaves for which a regression predecessor has already been found and avoid unnecessary traversal of the graph.

On the one hand, the propagation can result in saving some validation calls. On the other hand, the use of propagation may be in conflict with the desire to identify the latest regression predecessors. Therefore, the usage of propagation is optional. Section 4 demonstrates the behaviour of the algorithm both with and without the propagation step.

There are further three key aspects that affect the efficiency of the algorithm: the order in which leaves are chosen from the set UnprocessedLeaves, the method of building the path connecting a valid vertex with the invalid leaf, and the method of regression points identification. We focus on these three aspects in the following text. 


\subsection{Identification of Regression Points}

In this subsection we give the details of our solution to the problem of finding a regression point on a given path $p=\left\langle v_{0}, v_{1}, \ldots, v_{l}\right\rangle$ connecting a valid vertex $v_{0}$ with an invalid vertex $v_{l}$.

Linear search The simplest solution to the task is to evaluate the function valid for each vertex on the path, starting with $v_{l}$ and going backwards. As soon as a valid vertex $v_{i}$ is found, the algorithm outputs $\left(v_{i}, v_{i+1}\right)$ as a regression predecessor of $v_{l}$. By starting with $v_{l}$ and going backwards we guarantee that $\left(v_{i}, v_{i+1}\right)$ is the nearest regression point of $v_{l}$ along this path. The disadvantage of this approach is that in the worst case all vertices on the path are tested for validity. Because the commit graphs of VCS usually contain hundreds or thousands of commits and the evaluation of the function valid is assumed to be very expensive, the linear search is practically unusable.

Binary search Provided that the first vertex of the path is valid and the last is invalid (which is always our case) we can use binary search to find a regression point. Let $p=\left\langle v_{0}, v_{1}, \ldots v_{m i d}, \ldots, v_{l}\right\rangle$ be a path such that $v_{0}$ is valid, $v_{l}$ is invalid, and $v_{m i d}$ is the middle vertex of this path. If $v_{m i d}$ is valid then there is a regression point on the path $\left\langle v_{m i d}, \ldots, v_{l}\right\rangle$. Otherwise, there is a regression point on the path $\left\langle v_{0}, \ldots v_{m i d}\right\rangle$. We can thus always reduce $p_{l}$ into half and recursively repeat the procedure.

Contrary to the linear search approach it is not guaranteed that the binary search finds a regression point which is nearest to $v_{l}$. The main advantage of the binary search is that it evaluates the function valid only for logarithmically many vertices on the path $p$, which is optimal.

Multiplying search The so-called multiplying search approach combines the advantages of both binary and linear search approaches as it performs asymptotically fewer validity checks than the linear search and at the same time tends to find a regression point which is closer to the last vertex $v_{l}$ than the regression point found by the binary search.

Let $p=\left\langle v_{0}, v_{1}, \ldots, v_{l}\right\rangle$ be a path such that $v_{0}$ is valid and $v_{l}$ is invalid. The multiplying search first evaluates the function valid for the vertex $v_{l-1}$. If $v_{l-1}$ is not valid, then the function is stepwise evaluated for vertices $v_{l-2^{0}-2^{1}}, v_{l-2^{0}-2^{1}-2^{2}}$, $v_{l-\left(2^{4}-1\right)}, \ldots$ forming exponentially large gaps between individual vertices. The procedure eventually finds an $i$ such that the vertex $v_{l-\left(2^{i-1}-1\right)}$ is invalid and either $v_{l-\left(2^{i}-1\right)}$ is valid or $l-\left(2^{i}-1\right)<0$. If the former happens, then the procedure recursively continues with the new path $\bar{p}=\left\langle v_{l-\left(2^{i}-1\right)}, v_{l-\left(2^{i}-2\right)}, \ldots\right.$, $\left.v_{l-\left(2^{i-1}-1\right)}\right\rangle$. In the latter case the procedure recursively continues with the path $\bar{p}=\left\langle v_{0}, v_{1}, \ldots, v_{l-\left(2^{i-1}-1\right)}\right\rangle$. The procedure converges to a path containing only two vertices such that the first vertex of the path is valid and the second invalid, i.e., a regression point is found. For the complete description see Algorithm 3

The number $C(n)$ of vertices on which the function valid is evaluated on a path of length $n$ is bounded by the recurrence equation $C(n) \leq C\left(\frac{n}{2}\right)+\log n$. In 


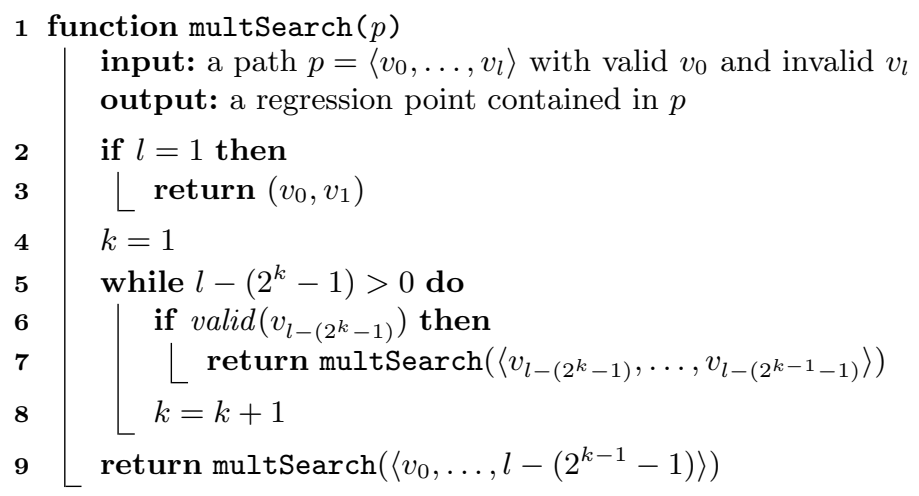

Algorithm 3: multiplying search

each recursive call the number of evaluations is at most $\log n$ and the length of the path is decreased at least by half. The solution of the recurrence equation (using the Master theorem [20]) gives an upper bound $\mathcal{O}\left(\log ^{2} n\right)$ on the number of vertices on which the function valid is evaluated.

\subsection{Leaf Selection and Path Construction}

Our next goal is to specify the order in which unprocessed leaves are chosen and determine the method of building a path connecting a valid vertex with the chosen leaf.

We assume that the directed acyclic graphs induced by VCS are represented using adjacency lists (see [6]) in which every vertex is equipped both with a list of its direct successors and a list of its direct predecessors. In the initialisation phase of RPA we compute the length of the shortest paths from $v$ to $l$ for each vertex $v \in V$ and invalid leaf $l \in L$. For every pair $(v, l) \in V \times L$ we maintain a successor of $v$ so that the chain of successors originating at the vertex $v$ runs forward along a shortest path from $v$ to $l$. This computation is done by running a backwards breadth-first search from each $l \in L$ using the list of predecessors, for details see e.g. [6].

In what follows we use $\operatorname{dist}(v, l)$ to denote the length of the shortest path leading from the vertex $v$ to the leaf $l$; we further define:

$$
\begin{aligned}
\operatorname{dist}(l)= & \min \{\operatorname{dist}(u, l) \mid u \in \text { KnownValid }\} \\
\operatorname{start}(l)= & u \text { such that } u \in \text { KnownValid } \\
& \text { and } \operatorname{dist}(u, l)=\operatorname{dist}(l) .
\end{aligned}
$$

In other words, $\operatorname{dist}(l)$ denotes the length of a shortest path leading to $l$ from a vertex $u$ for which the function valid has been evaluated and is valid (i.e. belongs to the set KnownValid). The first vertex of such a path is denoted $\operatorname{start}(l)$. As the set KnownValid changes during the computation, so may the values $\operatorname{dist}(l)$ 


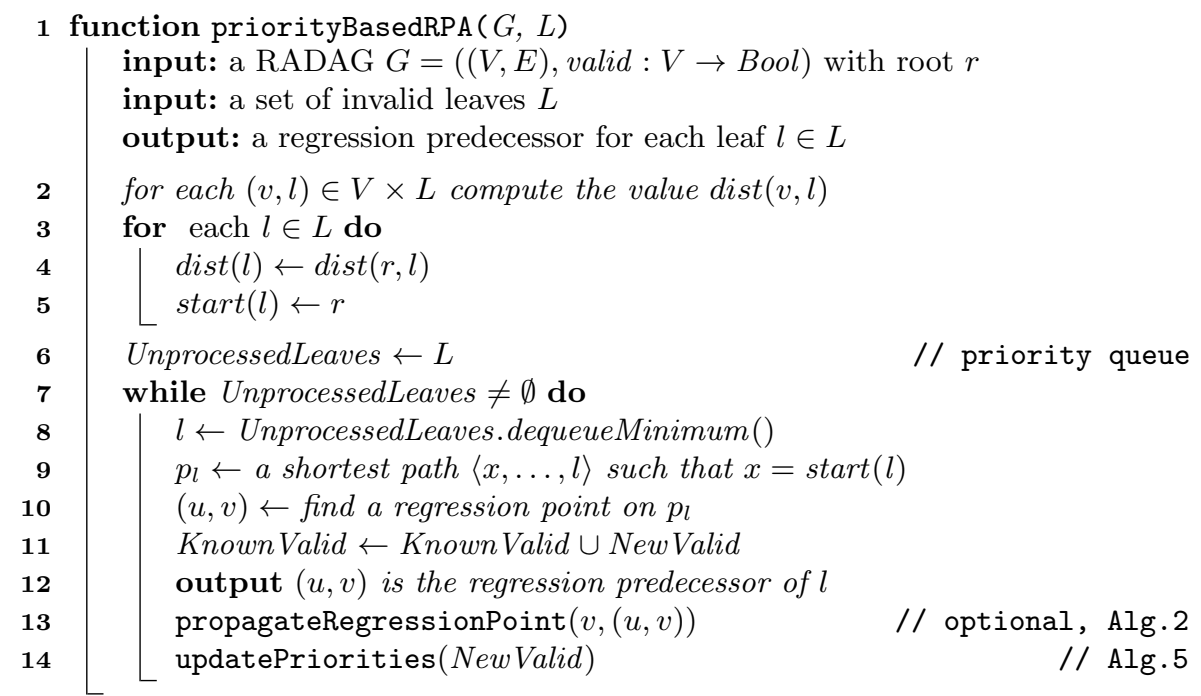

Algorithm 4: Regression Predecessor Algorithm

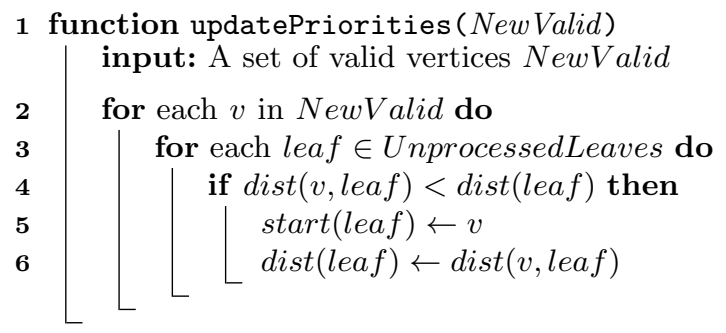

Algorithm 5: priority update

and $\operatorname{start}(l)$. Initially, only the root $r$ of the graph is known to be valid, therefore $\operatorname{dist}(l)=\operatorname{dist}(r, l)$ and $\operatorname{start}(l)=r$ for each $l \in L$.

The way in which RPA fixes the order in which invalid leaves are processed and determines which paths should be used for identification of regression points is based on the following observation. The shorter path we process the fewer number of evaluations of the function valid is performed, independent on the regression finding approach. For a complete description of the RPA algorithm see Algorithm 4 for an illustrative example see Sec. 3.4 .

The RPA algorithm maintains the set UnprocessedLeaves as a priority queue where each $l \in$ UnprocessedLeaves is assigned the priority dist(l). In every iteration the algorithm extracts the leaf $l$ with minimum priority from UnprocessedLeaves and constructs the shortest path leading to $l$. Moreover, each iteration is supplemented by the method updatePriorities (newValid) that updates the dist $(l)$ and $\operatorname{start}(l)$ values (see Algorithm 5). 


\subsection{Example}

\section{I. iteration}

- Removed vertices $=\emptyset$

- KnownValid $=\{a\}$

- Priority queue $=\langle p, g, k, m\rangle$

$-\operatorname{dist}(p)=5, \operatorname{dist}(g)=6$,

$\operatorname{dist}(k)=6, \operatorname{dist}(m)=7$

$-\operatorname{start}(p)=a$

- path $p_{p}=\langle a, b, c, n, o, p\rangle$

- Tested vertices: $o, c, n$

- Regression point of $p_{p}=(n, o)$

- Propagated to: $\{p\}$

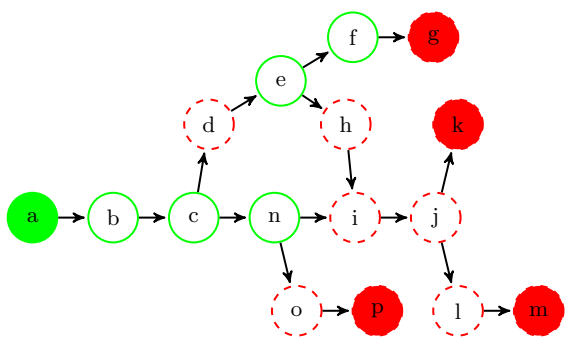

\section{II. iteration}

- Removed vertices $=\{o, p\}$

- KnownValid $=\{a, c, n\}$

- Priority queue $=\langle k, m, g\rangle$

$-\operatorname{dist}(k)=3, \operatorname{dist}(m)=4, \operatorname{dist}(g)=4$

$-\operatorname{start}(k)=n$

- path $p_{k}=\langle n, i, j, k\rangle$

- Tested vertices: $j, i$

- Regression point of $p_{k}=(n, i)$

- Propagated to: $\{k, m\}$

III. iteration

- Removed vertices $=\{o, p, i, j, k, l, m\}$

- KnownValid $=\{a, c, n\}$

- Priority queue $=\langle g\rangle$

$-\operatorname{dist}(g)=4$

$-\operatorname{start}(g)=c$

- path $p_{g}=\langle c, d, e, f, g\rangle$

- Tested vertices: $f$

- Regression point of $p_{g}=(f, g)$

- Propagated to: $\{g\}$

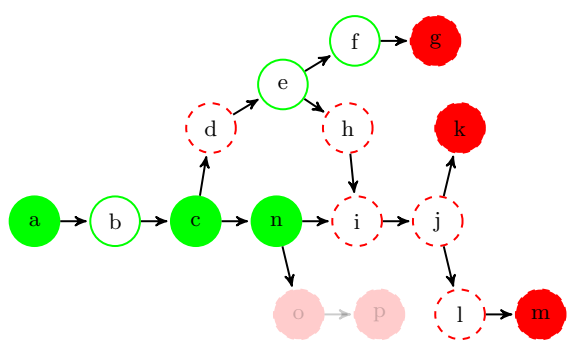

Fig. 2. An illustrative example

Figure 2 demonstrates the execution of RPA with multiplying search and propagation. In this example, the RADAG has only invalid leaves, $L=\{g, k, m, p\}$, and the task is to find the regression predecessors for all leaves. The computation consists of 3 iterations. The function valid is evaluated only for 7 out of 16 vertices and 3 regression points are found. We list the values of control variables in each iteration and illustrate them on the graph. The vertices on which the valid function has been evaluated are filled with green or red color depending on their validity. The vertices removed from the graph are shaded. 


\subsection{Related Work}

To the best of our knowledge, the first tool for finding regression points was the git bisect tool [10] which is a part of the distributed VCS git [8]. The method used in the git bisect tool is called bisection and it was subsequently adopted by other VCS like Mercurial [14, Subversion [16, and Bazaar [4].

The bisection algorithm takes as an input a single invalid commit and finds a regression point that precedes this commit. We give only a brief description of the algorithm here, more elaborated description can be found in 9 . The algorithm represents the commits using a directed acyclic graph and assumes that the function valid is monotone, i.e. that every successor of an invalid commit is also invalid. It starts by taking as an input a single invalid commit called "bad" together with a one or more commits which are known to be valid. Then, it iteratively repeats the following steps:

(i) Keep only the commits that: a) precede "bad" commit (including the "bad" commit itself) and b) do not precede a commit which is known to be valid (excluding the commits which are known to be valid).

(ii) Associate to each commit $c$ a number $r=\min \{(x+1), n-(x+1)\}$ where $x$ is the number of commits that precede the commit $c$ and $n$ is the total number of commits in the graph. Roughly speaking, this number represents the amount of information that can be obtained by evaluating the function valid on $c$. If $c$ is valid then all of its predecessors are valid (based on the assumption that the function valid is monotone), otherwise all of its successors are invalid.

(iii) Evaluate the function valid for the commit $v$ with the highest associated number. If $v$ is invalid then it becomes the "bad" commit.

Eventually there will be only one invalid commit left in the graph with one of its predecessors in the original graph being valid. This pair of vertices forms the regression predecessor of the original "bad" commit. Although the main idea of the bisection method is based on the monotonicity of the function valid, it is guaranteed that the algorithm finds a regression predecessor of the "bad" commit even if the function valid is not monotone.

There are two main drawbacks of git bisect comparing to RPA. First, the bisection algorithm does not tend to find the latest regression predecessor. Second, experiments (see the following section) demonstrate that git bisect evaluates more commits than RPA. The reason of this behaviour is that RPA prefers shortest paths while git bisect prefers vertices with the highest associated number. To demonstrate the difference let us consider a graph with one leaf and two paths connecting the root with the leaf. If one path is very short and the second one very long, then RPA prefers the short path while git bisect evaluates vertices on the long one. If a graph contains only one path leading to an invalid leaf, git bisect evaluates the same vertices as RPA combined with binary search.

There is also further related work that deals with problems similar to ours. Heuristics for automated culprit finding [22] are used for isolating one or more code changes which are suspected of causing a code failure in a sequence of project versions. They assume that the codebase is tested/validated regularly 
(e.g. after every $n$ commits) using some test suit. If a bug is detected, they search for the culprit only among the changes to the codebase that have been made since the latest appliance of the test suite. The individual versions are rated according to their potential to cause the failure (e.g. versions with many code changes are rated higher) and versions with high rate are tested as first. The culprit finding technique 22] is efficiently applicable only for searching in a short term history and it assumes that there is only one culprit.

Delta debugging 21 is a methodology to automate the debugging of programs using the approach of a hypothesis-trial-result loop. For a given code and a test case that detects a bug in the code, the delta debugging algorithm can be used to trim useless functions and lines of the code that are not needed to reproduce to bug. The delta debugging cannot be used for finding regression points in VCS. However, we believe that it can be incorporated into RPA and improve its performance by reducing the portion of code that need to be validated by the function valid.

A regression testing [1] and continuous integration testing [7] are types of software testing that verifies that software previously developed and tested still performs correctly even after it was changed or interfaced with other software. These two techniques are suitable for fixing bugs that are detected right after they are introduced. However, if a bug that lied in a codebase for some time is detected, e.g. because of extending the coverage of the tests, a technique like RPA need to be used. That is, RPA and regression testing/continous integration testing are mutually orthogonal techniques

SZZ 1813 is an algorithm for identifying commits in a VCS that introduced bugs, however it works in a quite different settings. It assumes, that the bug has been already fixed and that the commit that fixed the bug is explicitly known or can be found in a log file. This allows to identify particular lines of code that fixed the bug and this information is then exploited while searching for the bug-introducing commit. In our settings, the bugs are not fixed yet, thus SZZ cannot be used.

Finally, we relate the regression predecessors problem with well known problems from graph theory. The latest regression point can be found using the breadth-first-search (BFS) algorithm[12. As our goal is to minimize the number of validity queries, BFS is not suitable as it queries every vertex. Therefore, we come with a new, specialized, algorithm.

\section{Experimental Results}

We demonstrate the performance of the variants of RPA on two types of use cases. We first focus on the problem of finding a regression predecessor of a single invalid leaf. We then focus on the problem of finding regression predecessors of a set of invalid leaves. We also compare the performance of the RPA variants to that of the git bisect tool [109].

As benchmarks we use large real open source projects, taken from the GitHub open source showcases [11, with at least 8 active branches or at least 1000 


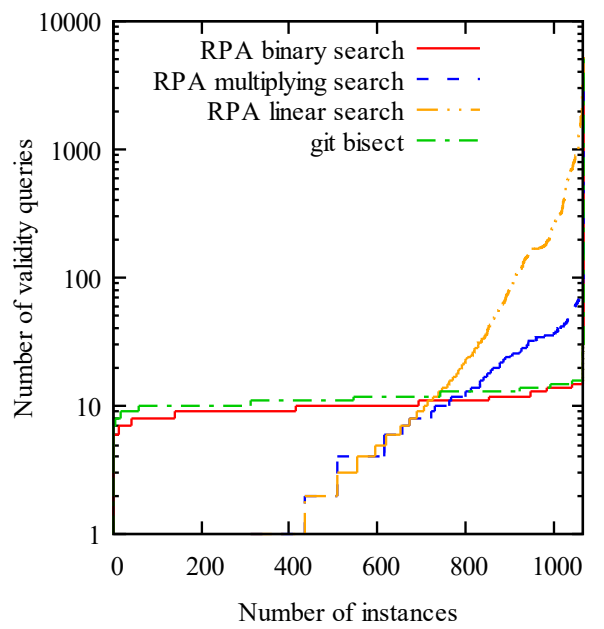

Fig. 3. Cumulative distribution plot of performed validity queries for each evaluated algorithm. A point with coordinates $[\mathrm{x}, \mathrm{y}]$ can be read as " $x$ instances were solved by using at most $y$ validity queries".

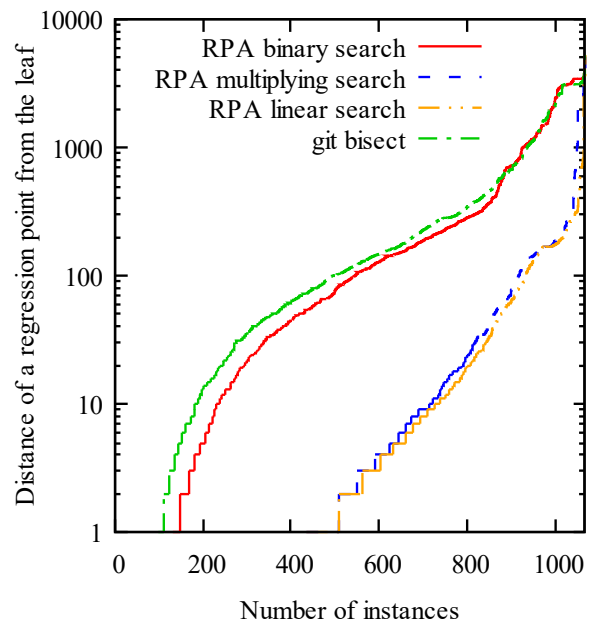

Fig. 4. Cumulative distribution plot of distance between regression predecessor and corresponding invalid leaf for each evaluated algorithm. A point with coordinates $[\mathrm{x}, \mathrm{y}]$ can be read as "in $x$ instances the distance was at most $y$ ".

commits. Due to the size of the projects it would be intractable to build and test all commits in these projects. Therefore we use those projects from [11] that employ TravisCI [19]. Travis CI is a service used to build and test projects hosted at GitHub and the results of all tests that were run on these projects are publicly available. Whenever our algorithm needs to validate a commit, it acquires the results of the tests from the publicly available Travis CI database instead. Overall we selected 84 projects with 1069 invalid leaves in total. Our selection includes for example the Rails web-application framework [17/3, the PHP Interpreter [15], or the ArangoDB [2].

We do not provide any details about the architecture of the computer on which we run the experiments because the computation time is not a relevant criterion in our study. It took a few seconds to run all the experiments because we didn't actually run the tests. As a main criterion for measuring the efficiency of evaluated algorithms we use the number of performed validity queries and the distance between identified regression points and corresponding invalid leaves (in order to measure the tendency to find the latest regression points). Complete results of all measurements are available at https://tinyurl.com/y857h82g.

\subsection{Single Invalid Leaf Instances}

We first analyse how variants of RPA and git bisect perform while searching for a regression predecessor of a single invalid leaf. In the case of finding a regression predecessor of a single invalid leaf, it makes no sense to use propagation. Therefore, 


\begin{tabular}{c|c|c|c}
$<$ & mult & bin & git \\
\hline mult & - & $736(69 \%)$ & $759(71 \%)$ \\
\hline bin & $313(29 \%)$ & - & $824(77 \%)$ \\
\hline git & $294(28 \%)$ & $25(2 \%)$ & -
\end{tabular}

Table 1. The number of instances on which the algorithm named in the row performed strictly less validity queries than the other algorithms.

\begin{tabular}{c|c|c|c}
$<$ & mult & bin & git \\
\hline mult & - & $717(67 \%)$ & $843(79 \%)$ \\
\hline bin & $26(2 \%)$ & - & $511(48 \%)$ \\
\hline git & $27(3 \%)$ & $328(30 \%)$ & -
\end{tabular}

Table 2. The number of instances on which the algorithm named in the row found a strictly closer regression predecessor than the other algorithms.

we always build the shortest path from a valid vertex and employ either binary or multiplying search. We also include the naive linear search approach that builds a path and checks one by one individual commits on the path.

The results comparing the number of the performed validity queries are shown in Fig. 3. In this plot, we show the cumulative distributions of the performed validity queries for each evaluated algorithm. The performance of git bisect and binary search was quite stable on all instances, they needed to perform at most 17 and 16 validity queries, respectively, to solve the hardest instances. On the other hand, the performance of multiplying search was less stable, it needed to perform from 1 to 101 validity queries. Linear search was negligibly better than exponential search on instances where the regression point was quite close, however it needed to perform up to 3458 validity queries on the harder instances.

In addition to the plot of cumulative distribution we show in Table 1 the number of instances on which one algorithm performed strictly less validity queries than its competitors. The table shows that binary search was superior to git bisect on most of the instances. This is caused by the nature of these two algorithms. Both of them need to perform just logarithmically many validity queries, but git bisect searches the whole graph whereas binary search traverses only a single path. Moreover, RPA always chooses the shortest usable path. Multiplying search can execute less than logarithmically many validity queries but it can also execute more than logarithmically many validity queries. Its performance depends on the position of regression points on the path. The closer the regression points are to the leaf the more likely the multiplying search approach outperforms the binary search approach (and thus also git bisect).

Besides the number of performed validity queries, we also measure the tendency of the algorithms to find the latest regression predecessors. So far we have not precisely defined which regression predecessor is the latest one and there is more than one suitable definition. In the case of binary, multiplying, and linear search we look for a regression predecessor on a path; thus, we can say that the latest regression predecessor is the regression point which is closest to the end of the path (i.e., closest to the leaf). In our experiments, multiplying search found the closest regression predecessor on the path in 86 percent of instances whereas binary search only in 29 percent of instances. This notion of latest regression predecessor is not applicable to git bisect because git bisect does not operate on paths. In order to compare RPA with git bisect, we measured 


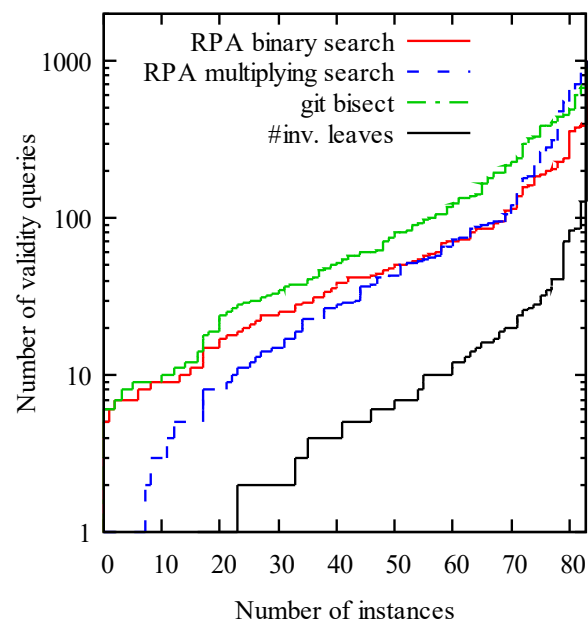

Fig. 5. Cumulative distributions of number of performed validity queries for git bisect and variants of RPA without propagation. The black line is the cumulative distribution of number of invalid leaves.

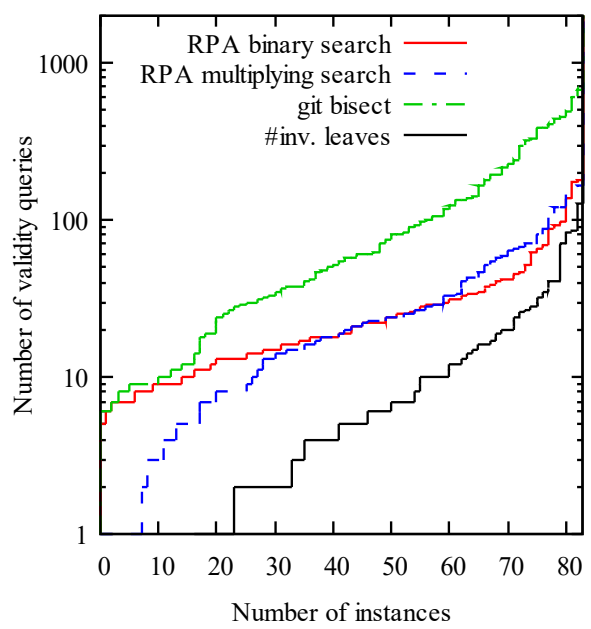

Fig. 6. Cumulative distributions of number of performed validity queries for git bisect and variants of RPA with propagation. The black line is the cumulative distribution of number of invalid leaves.

the distance between the found regression predecessor and the corresponding invalid leaf, i.e. the shortest path between these two vertices in the commit graph. Fig. 4 shows a plot of cumulative distributions of distance between regression predecessors and corresponding leaves for each evaluated algorithm. The results demonstrate that multiplying search substantially outperforms binary search and git bisect, and that binary search is slightly better than git bisect. The naive linear search is just negligibly better than multiplying search.

In addition, Table 2 shows the number of instances on which one algorithm found strictly closer regression point than its competitors. The multiplying search strictly dominates both its competitors; the regression predecessor found by multiplying search was closer to the leaf than the one found by git bisect in 79 percent of instances. Binary search performed slightly better, it was dominated by multiplying search only in 67 percent of instances.

\subsection{Sets of Invalid Leaves}

We now demonstrate the performance of the RPA variants on the problem of finding regression predecessors for a set of invalid leaves. In particular, we evaluate both proposed approaches for finding regression points, i.e., the binary and multiplying search. Moreover, we evaluate both these approaches in two variants: with and without regression point propagation (the optional part of RPA).

We also compare the variants of RPA to the git bisect tool. As mentioned in Sec. 3.5. git bisect deals with the problem of finding a regression predecessor 
of a single invalid leaf. Therefore, in order to solve the problem of regression predecessors for a given set of invalid leaves $L$, git bisect has to be run once per each leaf from $L$. As all these runs are independent, it might happen that some commits are evaluated repeatedly. In order to avoid the repeated evaluations, we supplement git bisect with a cache saving the results of the previous evaluations. Thus, every commit is evaluated at most once.

As benchmarks we used the 84 projects from GitHub showcases; the goal was to find a regression predecessor for every invalid leaf in every project. In this part of experimental evaluation we focus solely on the number of performed validity queries. Figures 5 and 6 show the cumulative distribution plots of the performed validity queries for the variants of RPA with and without propagation, respectively. In both plots we also include the results achieved by git bisect. Moreover, we include the cumulative distribution of the number of invalid leaves (solid red line), i.e. a point with coordinates $[x, y]$ means that $x$ instances have at most $y$ invalid leaves.

In general, the regression point propagation significantly reduces the overall number of performed validity queries. Considering the difference in performance between variants of RPA with multiplying and binary search, respectively, we observe the same behaviour as in the case of finding regression predecessors for single invalid leaves. There are some instances on which multiplying search outperformed binary search, and some instances on which binary search outperformed multiplying search. Git bisect performed conclusively the worst of all evaluated algorithms.

\subsection{Recommendations}

We have presented several variants of the RPA algorithm and the experimental results show that the variants are in general incomparable. There is no variant that would beat all the others independently of the comparison criteria. In the case where user searches for a regression predecessor of a single invalid leaf it makes no sense to use propagation. We suggest the user to use multiplying search if she prefers finding the closest regression points, and to use binary search if she prefers minimizing the number of performed validity queries.

In the other case, where user searches for regression predecessors of several invalid leaves, it might be worth to use propagation. If the user prefers finding the closest regression points to minimizing the number of performed validity queries, we suggest her not to use regression point propagation and employ multiplying search. In the opposite case, when user focus mainly on minimizing the number of validity queries, we suggest her to use regression point propagation and employ binary search as it guarantees that only logarithmically many validity queries will be performed.

\section{Conclusion}

We present a new algorithm, called the Regression Predecessors Algorithm (RPA), for finding regression points in projects under version control. The algorithm has 
several variants, the choice of which depends on whether we prefer to minimise the number of validity queries or the latest regression point. We have experimentally compared the variants among themselves as well as against the state-of-the art tool git bisect. The results show that the variants of RPA are in general incomparable as there is no variant that would beat all others independently of the criteria. The main strength of RPA lies in the ability to minimise the number of validity queries while respecting the requirement to find the latest regression point. In all cases RPA is superior to the algorithm used in git bisect.

\section{References}

1. Hiralal Agrawal, Joseph Robert Horgan, Edward W. Krauser, and Saul London. Incremental regression testing. In ICSM, pages 348-357. IEEE Computer Society, 1993.

2. ArangoDB. https://github.com/arangodb/arangodb, 2017. Accessed: 2017-02-02.

3. Michael Bächle and Paul Kirchberg. Ruby on rails. IEEE Software, 24(6):105-108, 2007.

4. Bazaar. http://bazaar.canonical.com/, 2017. Accessed: 2017-02-02.

5. Edmund M. Clarke, Orna Grumberg, and Doron A. Peled. Model checking. MIT Press, 2001.

6. Thomas H. Cormen, Charles E. Leiserson, Ronald L. Rivest, and Clifford Stein. Introduction to Algorithms (3. ed.). MIT Press, 2009.

7. Paul M Duvall. Continuous integration. Pearson Education India, 2007.

8. Git. https://git-scm.com/, 2017. Accessed: 2017-02-02.

9. Git bisect algorithm overview. https://git-scm.com/docs/git-bisect-lk2009 html 2017. Accessed: 2017-02-02.

10. Git bisect documentation. https://git-scm.com/docs/git-bisect, 2017. Accessed: 2017-02-02

11. GitHub Showcases. https://github.com/showcases, 2017. Accessed: 2017-02-02.

12. Dieter Jungnickel. Graphs, networks and algorithms, volume 5 of algorithms and computation in mathematics, 1999.

13. Sunghun Kim, Thomas Zimmermann, Kai Pan, and E. James Whitehead Jr. Automatic identification of bug-introducing changes. In $A S E$, pages 81-90. IEEE Computer Society, 2006.

14. Mercurial. https://www.mercurial-scm.org/, 2017. Accessed: 2017-02-02.

15. PHP interpreter. https://github.com/php/php-src 2017. Accessed: 2017-02-02.

16. C. Michael Pilato, Ben Collins-Sussman, and Brian W. Fitzpatrick. Version control with subversion - the standard in open source version control. O'Reilly, 2008.

17. Ruby on Rails. https://github.com/rails/rails, 2017. Accessed: 2017-02-02.

18. Jacek Sliwerski, Thomas Zimmermann, and Andreas Zeller. When do changes induce fixes? ACM SIGSOFT Software Engineering Notes, 30(4):1-5, 2005.

19. Travis CI. https://travis-ci.org/, 2017. Accessed: 2017-02-02.

20. Rakesh M. Verma. A general method and a master theorem for divide-and-conquer recurrences with applications. J. Algorithms, 16(1):67-79, 1994.

21. Andreas Zeller. Yesterday, my program worked. today, it does not. why? In ESEC / SIGSOFT FSE, volume 1687 of Lecture Notes in Computer Science, pages 253-267. Springer, 1999.

22. Celal Ziftci and Vivek Ramavajjala. Heuristics for automated culprit finding, November 3 2015. US Patent 9,176,731. 